\title{
Variational Approaches to Characterize Weak Solutions for Some Problems of Mathematical Physics Equations
}

\author{
Irina Meghea \\ Department of Mathematical Methods and Models, Faculty of Applied Sciences, University Politehnica of Bucharest, \\ 060042 Bucharest, Romania \\ Correspondence should be addressed to Irina Meghea; i_meghea@yahoo.com
}

Received 10 November 2015; Accepted 3 January 2016

Academic Editor: Khalil Ezzinbi

Copyright (C) 2016 Irina Meghea. This is an open access article distributed under the Creative Commons Attribution License, which permits unrestricted use, distribution, and reproduction in any medium, provided the original work is properly cited.

This paper is aimed at providing three versions to solve and characterize weak solutions for Dirichlet problems involving the $p$ Laplacian and the $p$-pseudo-Laplacian. In this way generalized versions for some results which use Ekeland variational principle, critical points for nondifferentiable functionals, and Ghoussoub-Maurey linear principle have been proposed. Three sequences of generalized statements have been developed starting from the most abstract assertions until their applications in characterizing weak solutions for some mathematical physics problems involving the abovementioned operators.

\section{Introduction}

Obtaining and/or characterization of weak solutions for problems of mathematical physics equations involving $p$ Laplacian and $p$-pseudo-Laplacian is a subject matter previously discussed by the author through several approach methods in [1-6]. New similar results can be found by other authors, for instance, Amiri and Zivari-Rezapour in [7], El Khalil and Ouanan in [8], Rhouma and Sayeb in [9], Yoshida in [10]. The importance of these operators also devolves from their involvement in actual modelings of natural phenomena as thermal transfer by LanchonDucauquis, Tulita, and Meuris in [11] or glacier sliding or flow by Partridge in [12] and Pélissier in [13]. In this paper three methods are proposed following three sequences of results, starting from abstract statements and finishing with their application to find weak solutions of Dirichlet problems for $p$-Laplacian and for $p$-pseudo-Laplacian as well. In the first succession of assertions, two results of Ghoussoub from [14] have been generalized replacing the frame of Hilbert space by reflexive strictly convex Banach space and the condition imposed to the goal function to be of $C^{1}$-Fréchet class by the weaker condition to have a lower semicontinuous and Gâteaux differentiable function. These two theoretical statements were used, together with other results concerning Dirichlet problems for both cited generalized operators, in finding weak solutions for these problems. The second proposition sequence involves critical points for nondifferentiable functional. In this context, two results of Chang from [15] have been generalized changing the space $H^{1}(\Omega)$ in $W_{0}^{1, p}(\Omega)$ and introducing $\Delta_{p}$ and $\Delta_{p}^{s}$ in some problems formulated for $\Delta$ by Costa and Gonçalves in [16]. The last series of assertions starts from GhoussoubMaurey linear principle which is used here to characterize weak solutions for some mathematical physics equations. Moreover, the problems were discussed and solved using important findings for these operators obtained by the author in $[5,6]$ in connection with specific properties of the Sobolev spaces involved.

\section{Critical Points and Weak Solutions for Elliptic Type Equations}

2.1. Theoretical Support. In order to introduce the first result, a theoretical support will be given starting with the following.

Ekeland Principle (see $[1,17,18])$. Let $(X, d)$ be a complete metric space and $\varphi: X \rightarrow(-\infty,+\infty]$ bounded from below, lower semicontinuous, and proper. For any $\varepsilon>0$ and $u$ of $X$ with

$$
\varphi(u) \leq \inf \varphi(X)+\varepsilon
$$


and for any $\lambda>0$, there exists $v_{\varepsilon}$ in $X$ such that

$$
\begin{aligned}
& \varphi\left(v_{\varepsilon}\right)<\varphi(w)+\frac{\varepsilon}{\lambda} d\left(v_{\varepsilon}, w\right) \quad \forall w \in X \backslash\left\{v_{\varepsilon}\right\}, \\
& \varphi\left(v_{\varepsilon}\right) \leq \varphi(u), \text { and } d\left(v_{\varepsilon}, u\right) \leq \lambda .
\end{aligned}
$$

Definition 1. Let $X$ be a real normed space, $\beta$ be a bornology on $X$, and let $\varphi: X \rightarrow \mathbf{R}$. Let $c$ be in $\mathbf{R}$ and $F$ a nonempty subset of $X . \varphi$ verifies the Palais-Smale condition on the level $c$ around $F$ (or relative to $F$ ), (PS) $)_{c, F}$, with respect to $\beta$, when $\forall\left(u_{n}\right)_{n \geq 1}$ a sequence of points in $X$ for which

$$
\begin{array}{r}
\lim _{n \rightarrow \infty} \varphi\left(u_{n}\right)=c, \\
\lim _{n \rightarrow \infty}\left\|\nabla_{\beta} \varphi\left(u_{n}\right)\right\|=0, \\
\lim _{n \rightarrow \infty} \operatorname{dist}\left(u_{n}, F\right)=0,
\end{array}
$$

this sequence has a convergent subsequence.

To clarify the above notation, let $\beta$ be a bornology on $X$ and let $f: X \rightarrow \overline{\mathbf{R}}$ locally finite in the point $a$. By definition $f$ is $\beta$-differentiable in $a$, if there exists $\varphi$ in the dual $X^{*}$ such that for every $S$ in $\beta$ we have $\lim _{t \rightarrow 0, h \in S} u((f(a+t h)-$ $f(a)) / t$ ) $=\varphi(h)$ (uniform limit on $S$ for $t \rightarrow 0$ ). $\varphi$ is the $\beta$ derivative of $f$ in $a$, and it is denoted by $\nabla_{\beta} f(a)$.

Through the minimization on $F$ of a functional (minimization with constraints) global critical points of this may be obtained.

As a preliminary, we generalize some results from [14] introducing Banach space instead of Hilbert space and the Gâteaux differentiability instead of $C^{1}$-class Fréchet.

Proposition 2. Let $X$ be a real reflexive strictly convex Banach space, let $\varphi: X \rightarrow \mathbf{R}$ be lower semicontinuous and Gâteaux differentiable and let $F$ be a closed subset of $X$ such that for every $u$ from $F$ with the metric gradient $\nabla \varphi(u) \neq 0$, for sufficiently small $r>0$,

$$
\left(u-\delta \frac{\nabla \varphi(u)}{\|\nabla \varphi(u)\|}\right) \in F, \quad \forall \delta \in[0, r] .
$$

Then, if $\varphi$ is lower bounded on $F$, for every $\left(v_{n}\right)_{n \geq 1} a$ minimizing sequence for $\varphi$ on $F$, there exists a sequence $\left(u_{n}\right)_{n \geq 1}$ in F such that

$$
\begin{aligned}
\left\|\varphi^{\prime}\left(u_{n}\right)\right\| & \leq \sqrt{\varepsilon_{n}}, \\
\varphi\left(u_{n}\right) & \leq \varphi\left(v_{n}\right) \quad \forall n, \\
\lim _{n \rightarrow \infty}\left\|u_{n}-v_{n}\right\| & =0,
\end{aligned}
$$

where $\varepsilon_{n}>0$ and $\varepsilon_{n} \rightarrow 0$.

Remark 3. This result is reported in [14] as Lemma 9 in the frame of the Hilbert spaces having the function $\varphi$ of $C^{1}$-class Fréchet, but condition (5) is more complicated due to another condition imposed to the set $F$.
Explanations. Let us introduce the definition of the metric gradient in order to provide other observations relative to this central notion for this statement. In a real normed space $X$, consider the Gâteaux differentiable functional $f: X \rightarrow \mathbf{R}$. The metric gradient of $f$ is the multiple-valued mapping $\nabla f$ : $X \rightarrow \mathscr{P}(X), \nabla f(x)=i^{-1} J_{*} f_{w}^{\prime}(x)$, where $J_{*}: X^{*} \rightarrow \mathscr{P}\left(X^{* *}\right)$ is the duality mapping on $X^{*}$ corresponding to the identity and $i$ the canonical injection of $X$ into $X^{* *}: i(x)=x^{* *}$, $\left\langle x^{* *}, x^{*}\right\rangle=\left\langle x^{*}, x\right\rangle, \forall x^{*} \in X^{*}$. Consequently, for any $x \in X: \nabla f(x)=\left\{y \in X: i(y) \in J_{*} f_{w}^{\prime}(x)\right\}=\{y \in$ $X:\left\langle i(y), f_{w}^{\prime}(x)\right\rangle=\left\langle f_{w}^{\prime}(x), y\right\rangle=\left\|f_{w}^{\prime}(x)\right\|^{2},\|i(y)\|=\|y\|=$ $\left.\left\|f_{w}^{\prime}(x)\right\|\right\}$. If $X$ is reflexive, for any $x \in X, \nabla f(x)$ is nonempty. $X^{* *}$ being strictly convex, $J_{*}$ is single-valued. So, if $X$ is reflexive and strictly convex, then $\nabla f: X \rightarrow X, \nabla f(x)=$ $i^{-1} J_{*} f_{w}^{\prime}(x)$, and the following equalities hold:

$$
\begin{aligned}
\left\langle f_{w}^{\prime}(x), \nabla f(x)\right\rangle & =\left\|f_{w}^{\prime}(x)\right\|^{2}, \\
\|\nabla f(x)\| & =\left\|f_{w}^{\prime}(x)\right\| .
\end{aligned}
$$

Go now to the proof of Proposition 2.

Proof. Denote $c:=\inf \varphi(F)$ and let $n$ be from N. For $\varepsilon_{n}:=$ $\varphi\left(v_{n}\right)-c+1 / n$, hence $\varepsilon_{n}>0$, we have $\varphi\left(v_{n}\right)<c+\varepsilon_{n}$. Apply the enunciated Ekeland principle with $\lambda=\sqrt{\varepsilon_{n}}, \exists u_{n}$ in $F$ with known properties. Thus we obtain the sequence $\left(u_{n}\right)_{n \geq 1}$ satisfying (6), (7) $\left(\left\|u_{n}-v_{n}\right\| \leq \sqrt{\varepsilon_{n}}, \varepsilon_{n} \rightarrow 0\right)$, and

$$
\varphi(v) \geq \varphi\left(u_{n}\right)-\sqrt{\varepsilon_{n}}\left\|v-u_{n}\right\| \quad \forall v \in F .
$$

Verify (5). It is sufficient to work under the assumption $\left\|\varphi_{w}^{\prime}\left(u_{n}\right)\right\|>0 \forall n$. Thus apply the hypothesis made in the statement with respect to $F$ with $u=u_{n}$ and denoting, for $\delta \in(0, r], v_{\delta}:=u_{n}-\delta\left(\nabla \varphi\left(u_{n}\right) /\left\|\nabla \varphi\left(u_{n}\right)\right\|\right)(\epsilon F)$, replace $v_{\delta}$ in (9) and find,

$$
\sqrt{\varepsilon_{n}}\left\|v_{\delta}-u_{n}\right\| \geq \varphi\left(u_{n}\right)-\varphi\left(v_{\delta}\right),
$$

multiply this inequality by $1 / \delta, \delta>0$, and take the limit for $\delta \rightarrow 0+$ in order to keep the sense of the inequality. Remark that $\lim _{\delta \rightarrow 0} v_{\delta}=u_{n} ; \lim _{\delta \rightarrow 0}\left(\left\|v_{\delta}-u_{n}\right\| / \delta\right)=$ $\lim _{\delta \rightarrow 0}\left(\left(\delta\left\|\nabla \varphi\left(u_{n}\right)\right\| /\left\|\nabla \varphi\left(u_{n}\right)\right\|\right) / \delta\right)=1$. Consider that the existence of the limit for $\delta \rightarrow 0$ implies the existence of the limit for $\delta \rightarrow 0 \pm$ together with their equality, $\lim _{\delta \rightarrow 0+}\left(\left(\varphi\left(u_{n}\right)-\varphi\left(v_{\delta}\right)\right) / \delta\right)=$ $\lim _{\delta \rightarrow 0+}\left(\left(\varphi\left(u_{n}-\delta \nabla \varphi\left(u_{n}\right) /\left\|\nabla \varphi\left(u_{n}\right)\right\|\right)-\varphi\left(u_{n}\right)\right) /-\delta\right)=$ $\varphi_{w}^{\prime}\left(u_{n}\right)\left(\nabla \varphi\left(u_{n}\right) /\left\|\nabla \varphi\left(u_{n}\right)\right\|\right)=\left(1 /\left\|\nabla \varphi\left(u_{n}\right)\right\|\right)\left\langle\varphi_{w}^{\prime}\left(u_{n}\right), \nabla \varphi\left(u_{n}\right)\right\rangle$ $=\left(1 /\left\|\nabla \varphi\left(u_{n}\right)\right\|\right)\left\|\varphi_{w}^{\prime}\left(u_{n}\right)\right\|^{2}=\left\|\varphi_{w}^{\prime}\left(u_{n}\right)\right\|$, taking into account the definition of the Gâteaux derivative and the above considerations on the metric gradient, and (5) is also fulfilled.

Remark 4. The Gâteaux derivative from the above statement can be replaced by any $\beta$-derivative and the result remains the same. In the case of the Fréchet derivative, it must remove the condition " $\varphi$ lower semicontinuous" from the statement.

Notation 1. $\varphi: X \rightarrow \mathbf{R} \beta$-differentiable; $c \in \mathbf{R} \Rightarrow$

$$
K_{c}(\varphi):=\left\{x \in X: \varphi(x)=c, \nabla_{\beta} \varphi(x)=0\right\} .
$$


Proposition 5. Let $X$ be a real reflexive strictly convex Banach space and $\varphi: X \rightarrow \mathbf{R}$ lower semicontinuous and Gâteaux differentiable and $F$ is a nonempty convex closed subset such that $(I-\nabla \varphi)(F) \subset F, I$ the identity map. If $\varphi$ is lower bounded on $F$, then for every $\left(v_{n}\right)_{n \geq 1}$ a minimizing sequence for $\varphi$ on $F$, there is a sequence $\left(u_{n}\right)_{n \geq 1}$ in F such that

$$
\begin{aligned}
\varphi\left(u_{n}\right) & \leq \varphi\left(v_{n}\right) \quad \forall n, \\
\lim _{n \rightarrow \infty}\left\|u_{n}-v_{n}\right\| & =0, \\
\lim _{n \rightarrow \infty}\left\|\varphi_{w}^{\prime}\left(u_{n}\right)\right\| & =0 .
\end{aligned}
$$

Moreover, if $\varphi$ satisfies $(\mathrm{PS})_{c, F}$, where $c=\inf \varphi(F)$, then

$$
F \cap K_{c}(\varphi) \neq \varnothing \text {. }
$$

Proof. Applying Proposition 2, (4) is satisfied indeed: if $u \in F$ and $\varphi_{w}^{\prime}(u) \neq 0$, then, $F$ being convex,

$$
\begin{aligned}
u-\delta \frac{\nabla \varphi(u)}{\|\nabla \varphi(u)\|}= & \left(1-\frac{\delta}{\left\|\varphi_{w}^{\prime}(u)\right\|}\right) u \\
& +\frac{\delta}{\left\|\varphi_{w}^{\prime}(u)\right\|}(I-\nabla \varphi)(u) \in F .
\end{aligned}
$$

Let $\left(u_{n}\right)_{n \geq 1}$ be the sequence given by the statement. $c \leq$ $\varphi\left(u_{n}\right) \leq \varphi\left(v_{n}\right) \forall n$, hence $\varphi\left(u_{n}\right) \rightarrow c .\left\|\varphi_{w}^{\prime}\left(u_{n}\right)\right\| \stackrel{(5)}{\leq} \sqrt{\varepsilon_{n}}$, hence $\left\|\varphi_{w}^{\prime}\left(u_{n}\right)\right\| \rightarrow 0$, clearly dist $\left(u_{n}, F\right)=0$, and consequently $\left(u_{n}\right)_{n \geq 1}$ has a convergent subsequence $\left(u_{k_{n}}\right)_{n \geq 1}, u_{k_{n}} \rightarrow u_{0} \in F$. This implies $\left\|\varphi_{w}^{\prime}\left(u_{k_{n}}\right)\right\| \rightarrow\left\|\varphi_{w}^{\prime}\left(u_{0}\right)\right\|=0$, and $u_{0}$ is a global critical point of $\varphi$ contained in $F$.

2.2. Weak Solutions. Open set of $C^{1}$ class in $\mathbf{R}^{N}$. Use the notations (the norm is that Euclidean from $\mathbf{R}^{N-1}$ ): $\mathbf{R}_{+}^{N}=\{x=$ $\left.\left(x^{\prime}, x_{N}\right): x_{N}>0\right\}, Q=\left\{x=\left(x^{\prime}, x_{N}\right):\left\|x^{\prime}\right\|<1,\left|x_{N}\right|<1\right\}$, $Q_{+}=Q \cap \mathbf{R}_{+}^{N}, Q_{0}=\left\{x=\left(x^{\prime}, x_{N}\right):\left\|x^{\prime}\right\|<1, x_{N}=0\right\}$. Let $\Omega$ be an open nonempty set in $\mathbf{R}^{N}, \Omega \neq \mathbf{R}^{N}$, and $\partial \Omega$ its boundary. By definition, $\Omega$ is of $C^{1}$ class if $\forall x$ from $\partial \Omega \exists U$ a neighbourhood of $x$ in $\mathbf{R}^{N}$ and $f: Q \rightarrow U$ bijective such that $f \in C^{1}(\bar{Q}), f^{-1} \in C^{1}(\bar{U}), f\left(Q_{+}\right)=U \cap \Omega$, and $f\left(Q_{0}\right)=$ $U \cap \partial \Omega$. Weak solution. Let $\Omega$ be an open bounded nonempty set in $\mathbf{R}^{N}, N>1, f: \Omega \times \mathbf{R}^{N} \rightarrow \mathbf{R}$, and $u_{0} \in W_{0}^{1, p}(\Omega)$. Consider the problems

$$
\begin{aligned}
-\Delta_{p} u & =f(x, u), \quad x \in \Omega \\
u & =0 \quad \text { on } \partial \Omega, \\
-\Delta_{p}^{s} u & =f(x, u), \quad x \in \Omega \\
u & =0 \quad \text { on } \partial \Omega .
\end{aligned}
$$

Actually $u=u_{0}$ on $\partial \Omega$ means $u \mid \partial \Omega=u_{0}$, where $\gamma: u \rightarrow$ $u \mid \partial \Omega$ is the trace operator, a continuous linear mapping from $W^{1, p}(\Omega)$ in $\mathbf{L}^{p}(\partial \Omega), p \in[1,+\infty)$. We have $\gamma^{-1}(0)=$ $W_{0}^{1, p}(\Omega)\left(={\overline{C_{c}^{1}(\Omega)}}^{W^{1, p}(\Omega)}\right)$ and $u \in W^{1, p}(\Omega) \cap C(\bar{\Omega}) \Rightarrow \gamma(u)=$ $u \mid \partial \Omega \cdot \bar{u}$ from $X=W_{0}^{1, p}(\Omega)$ is by definition a weak solution for $(*)$ and $(* *)$, respectively, if $\bar{u}=0$ on $\partial \Omega$ and

$$
\begin{aligned}
& \int_{\Omega}|\nabla \bar{u}|^{p-2} \nabla \bar{u} \cdot \nabla v d x-\int_{\Omega} f(x, \bar{u}(x)) v d x=0 \\
& \qquad v v \in W_{0}^{1, p}(\Omega) \\
& \text { respectively } \sum_{i=1}^{N} \int_{\Omega}\left|\frac{\partial \bar{u}}{\partial x_{i}}\right|^{p-2} \frac{\partial \bar{u}}{\partial x_{i}} \frac{\partial v}{\partial x_{i}} d x \\
& -\int_{\Omega} f(x, \bar{u}(x)) v d x=0 \quad \forall v \in W_{0}^{1, p}(\Omega) .
\end{aligned}
$$

Remark 6. Here $\nabla w$ is the weak gradient, and it is equal to $\left(\partial w / \partial x_{1}, \ldots, \partial w / \partial x_{N}\right), \partial w / \partial x_{i}$ the weak derivatives; $|\nabla w|^{2}=\sum_{i=1}^{N}\left|\partial w / \partial x_{i}\right|^{2} . X:=W_{0}^{1, p}(\Omega)$ is endowed in the first case $(*)$ with the norm $\|\cdot\|_{1, p}$; that is, $\|u\|_{1, p}=$ $\|u\|_{W_{0}^{1, p}(\Omega)}=\|u\|_{L^{p}(\Omega)}+\sum_{i=1}^{N}\left\|\partial u / \partial x_{i}\right\|_{L^{p}(\Omega)}$, which is equivalent to the norm $u \rightarrow\left(\|u\|_{L^{p}(\Omega)}^{p}+\sum_{i=1}^{N}\left\|\partial u / \partial x_{i}\right\|_{L^{p}(\Omega)}^{p}\right)^{1 / p}$. For the second case $(* *)$, equip the same vector space with the norm $u \rightarrow \mid \mathbf{|} u \mathbf{|}_{1, p}=\left(\sum_{i=1}^{N}\left\|\partial u / \partial x_{i}\right\|_{\mathbf{L}^{p}(\Omega)}^{p}\right)^{1 / p}$, which is equivalent to $u \rightarrow|u|_{1, p}=\sum_{i=1}^{N}\left\|\partial u / \partial x_{i}\right\|_{L^{p}(\Omega)}$. Nemytskii Operator. Let be $\mathbf{R}^{N}, N \geq 1, \mu$ the Lebesgue measure in $\mathbf{R}^{N}, \Omega$ open nonempty Lebesgue measurable and $\mathscr{M}(\Omega):=\{u: \Omega \rightarrow$ $\mathbf{R} \mid u$ Lebesgue measurable $\}$. By definition $f: \Omega \times \mathbf{R} \rightarrow \mathbf{R}$ is a Carathéodory function if $f(\cdot, s)$ is Lebesgue measurable $\forall s \in \mathbf{R}$ and $f(x, \cdot)$ is continuous $\forall x \in \Omega \backslash A, \mu(A)=0$. In this case, for every $u$ from $\mathscr{M}(\Omega)$ one may consider the function $N_{f}: \mathscr{M}(\Omega) \rightarrow \mathscr{M}(\Omega), N_{f} u: N_{f} u(x)=f(x, u(x))$, Nemytskii operator. Suppose $\mu(\Omega)<+\infty$. Then $u_{n}(x) \underset{x \in \Omega}{\stackrel{\mu}{\longrightarrow}}$ $u_{0}(x) \Rightarrow N_{f} u_{n}(x) \underset{x \in \Omega}{\stackrel{\mu}{\longrightarrow}} N_{f} u_{0}(x)$. Assume that $f$ satisfies the growth condition: $|f(x, s)| \leq c|s|^{p-1}+b(x), \forall x \in \Omega \backslash A$ with $\mu(A)=0, \forall s \in \mathbf{R}$, where $c \geq 0, p>1$, and $b \in \mathbf{L}^{q}(\Omega)$, $q \in[1,+\infty]$.

Then $N_{f}\left(\mathbf{L}^{(p-1) q}(\Omega)\right) \subset \mathbf{L}^{q}(\Omega) ; N_{f}$ is continuous $(q<$ $+\infty)$ and bounded on $\mathbf{L}^{(p-1) q}(\Omega)$. If $\Omega$ is bounded and $1 / p+$ $1 / q=1$, then $N_{f}\left(\mathbf{L}^{p}(\Omega)\right) \subset \mathbf{L}^{q}(\Omega)$ with $N_{f}$ continuous; moreover, $N_{F}\left(\mathbf{L}^{p}(\Omega)\right) \subset \mathbf{L}^{1}(\Omega)$ with $N_{F}$ continuous (see, e.g., [2]), where $F(x, s)=\int_{0}^{s} f(x, t) d t$, and $\Phi: \mathbf{L}^{p}(\Omega) \rightarrow \mathbf{R}$, $\Phi(u)=\int_{\Omega} F(x, u(x)) d x$ is of Fréchet $C^{1}$ class and $\Phi^{\prime}=N_{f}$ [14], so it is also Gâteaux differentiable.

Theorem 7. Let $\Omega$ be an open bounded nonempty set in $\mathbf{R}^{N}$ and $f: \Omega \times \mathbf{R} \rightarrow \mathbf{R}$ a Carathéodory function with the growth condition:

$$
|f(x, s)| \leq c|s|^{p-1}+b(x)
$$

where $c>0,2 \leq p \leq 2 N /(N-2)$ when $N \geq 3$, and $2 \leq p<$ $+\infty$ when $N=1,2$, and where $b \in \mathbf{L}^{q}(\Omega), 1 / p+1 / q=1$. 
Then the energy functional $\varphi: W_{0}^{1, p}(\Omega) \rightarrow \mathbf{R}$,

$$
\varphi(u)=\frac{1}{p}\|u\|_{1, p}^{p}-\int_{\Omega} F(x, u(x)) d x
$$

for the problem $(*)$

$$
\text { respectively }
$$

$$
\varphi(u)=\frac{1}{p} \mid \mathbf{l} \mathbf{|}_{1, p}^{p}-\int_{\Omega} F(x, u(x)) d x
$$

for the problem $(* *)$,

where $F(x, s)=\int_{0}^{s} f(x, t) d t$ is Gâteaux differentiable on $W_{0}^{1, p}(\Omega) \backslash\{0\}$ and

$$
\begin{aligned}
& \varphi_{w}^{\prime}(u)(v)= \int_{\Omega}|\nabla u|^{p-2} \nabla u \cdot \nabla v d x \\
&-\int_{\Omega} f(x, u(x)) v d x \\
& \forall u, v \in W_{0}^{1, p}(\Omega) \text { respectively } \\
& \varphi_{w}^{\prime}(u)(v)=\sum_{i=1}^{N} \int_{\Omega}\left|\frac{\partial u}{\partial x_{i}}\right|^{p-2} \frac{\partial u}{\partial x_{i}} \frac{\partial v}{\partial x_{i}} d x \\
&-\int_{\Omega} f(x, u(x)) v d x=0 \\
& \quad \forall u, v \in W_{0}^{1, p}(\Omega) .
\end{aligned}
$$

Proof. One may consider $\varphi$, in both cases, as the sum of two other functions. The second of these functions being Gâteaux differentiable (see the above consideration), it is sufficient to remark that also the maps $u \rightarrow(1 / p)\|u\|_{1, p}^{p}$ and $u \rightarrow$ $(1 / p) \mathbf{I} \mathbf{l}_{1, p}^{p}$ are Gâteaux differentiable on $W_{0}^{1, p}(\Omega) \backslash\{0\}[2,19]$ and then $\varphi$ is Gâteaux differentiable on $W_{0}^{1, p}(\Omega) \backslash\{0\}$.

Corollary 8. Let $\Omega$ and $f$ be as in Theorem 7. Then the weak solutions of $(*)$ and $(* *)$, respectively, are precisely the critical points of the functional $\varphi: W_{0}^{1, p}(\Omega) \rightarrow \mathbf{R}:$

$$
\begin{aligned}
\varphi(u) & =\frac{1}{p}\|u\|_{1, p}^{p}-\int_{\Omega} F(x, u(x)) d x, \\
F(x, s) & :=\int_{0}^{s} f(x, t) d t \\
\text { respectively } \varphi(u) & =\frac{1}{p} \mid \mathbf{l} \mathbf{|}_{1, p}^{p}-\int_{\Omega} F(x, u(x)) d x, \\
F(x, s) & :=\int_{0}^{s} f(x, t) d t .
\end{aligned}
$$

Proof. Indeed, if $\bar{u}$ is a weak solution of $(*)$ and $(* *)$, respectively, then $\varphi_{w}^{\prime}(\bar{u})(v)=0 \forall v \in W_{0}^{1, p}(\Omega)((15)$ and (16), resp., Theorem 7); hence $\varphi_{w}^{\prime}(\bar{u})=0$. The inverse assertion is obvious.
Weak Subsolutions and Weak Supersolutions of $(*)$ and $(* *)$. Let $\Omega$ be an open bounded set of $C^{1}$ class in $\mathbf{R}^{N}, N \geq 3$, let $f$ : $\Omega \times \mathbf{R} \rightarrow \mathbf{R}$ be a Carathéodory function, and let $\bar{u} \in W_{0}^{1, p}(\Omega)$. $\bar{u}$ is a weak subsolution and a weak supersolution, respectively, of $(*)$ or $(* *)$ if

$$
\bar{u} \leq 0 \quad \text { on } \partial \Omega \text { respectively } \bar{u} \geq 0 \text { on } \partial \Omega \text {, and }
$$

$$
\begin{aligned}
\int_{\Omega}|\nabla \bar{u}|^{p-2} \nabla \bar{u} \cdot \nabla v d x \leq \int_{\Omega} & f(x, \bar{u}(x)) v d x \\
& \forall v \in W_{0}^{1, p}(\Omega), v \geq 0,
\end{aligned}
$$

respectively,

$$
\begin{aligned}
\int_{\Omega}|\nabla \bar{u}|^{p-2} \nabla \bar{u} \cdot \nabla v d x \geq \int_{\Omega} & f(x, \bar{u}(x)) v d x \\
& \forall v \in W_{0}^{1, p}(\Omega), \quad v \geq 0,
\end{aligned}
$$

or

$$
\begin{array}{r}
\sum_{i=1}^{N} \int_{\Omega}\left|\frac{\partial \bar{u}}{\partial x_{i}}\right|^{p-2} \frac{\partial \bar{u}}{\partial x_{i}} \frac{\partial v}{\partial x_{i}} d x \leq \int_{\Omega} f(x, \bar{u}(x)) v d x \\
\forall v \in W_{0}^{1, p}(\Omega), \quad v \geq 0
\end{array}
$$

respectively

$$
\begin{aligned}
\sum_{i=1}^{N} \int_{\Omega}\left|\frac{\partial \bar{u}}{\partial x_{i}}\right|^{p-2} \frac{\partial \bar{u}}{\partial x_{i}} \frac{\partial v}{\partial x_{i}} d x \geq & \int_{\Omega} f(x, \bar{u}(x)) v d x \\
& \forall v \in W_{0}^{1, p}(\Omega), \quad v \geq 0 .
\end{aligned}
$$

Proposition 9. Let $\Omega$ be an open bounded of $C^{1}$ class set in $\mathbf{R}^{N}, N \geq 3$ and $f: \Omega \times \mathbf{R} \rightarrow \mathbf{R}$ a Carathéodory function and $u_{1}, u_{2}$ from $W_{0}^{1, p}(\Omega)$ bounded weak subsolution and weak supersolution of $(*)$, respectively, with $u_{1}(x) \leq u_{2}(x)$ a.e. on $\Omega$. Suppose that $f$ verifies (17) and there is $\rho>0$ such that the function $g: g(x, s)=f(x, s)+\rho$ s is strictly increasing in $s$ on $\left[\inf u_{1}(\Omega)\right.$, sup $\left.u_{2}(\Omega)\right]$. Then there is a weak solution $\bar{u}$ of $(*)$ in $W_{0}^{1, p}(\Omega)$ with the property

$$
u_{1}(x) \leq \bar{u}(x) \leq u_{2}(x) \quad \text { a.e. on } \Omega .
$$

Proof. Take the equivalent norm on $X=W_{0}^{1, p}(\Omega)$ :

$$
\|u\|=\left(\rho\|u\|_{L^{p}(\Omega)}^{p}+\sum_{i=1}^{N}\left\|\frac{\partial u}{\partial x_{i}}\right\|_{L^{p}(\Omega)}^{p}\right)^{1 / p} .
$$

Consider the functional $\varphi: W_{0}^{1, p}(\Omega) \rightarrow \mathbf{R}:$

$$
\begin{aligned}
\varphi(u) & =\frac{1}{p}\|u\|^{p}-\int_{\Omega} G(x, u(x)) d x \\
G(x, s) & :=\int_{0}^{s} g(x, t) d t .
\end{aligned}
$$

$\varphi$ is Gâteaux differentiable and its critical points are the weak solutions of $(*)$ (see Corollary 8 ). $\varphi$ is also lower bounded, 
the norm on $\mathbf{L}^{p}(\Omega)$ actually being of Fréchet $C^{1}$ class (see, e.g., [20] or [21]). Use Proposition $5,(X,\|\cdot\|)$ being a reflexive strictly convex Banach space (see, e.g., [2]). Let be

$$
\begin{aligned}
\mathscr{F} & :=\left\{u \in W_{0}^{1, p}(\Omega): u_{1}(x) \leq u(x)\right. \\
& \left.\leq u_{2}(x) \text { a.e. on } \Omega\right\} .
\end{aligned}
$$

$\mathscr{F}$ is closed convex. We also get

$$
(I-\nabla \varphi) \mathscr{F} \subset \mathscr{F}
$$

Here $\nabla \varphi$ denotes the metric gradient of $\varphi$. Since $(X,\|\cdot\|)$ is reflexive and strictly convex (see, e.g., [2]), thus $\nabla \varphi$ is univaluated and it has the above described properties. Indeed, let $u$ be in $\mathscr{F}$ and $v:=(I-\nabla \varphi)(u)$. We should prove that $v \in \mathscr{F} \cdot v=u-\nabla \varphi(u) \in W_{0}^{1, p}(\Omega)$ and $u_{1}(x) \leq v(x) \leq$ $u_{2}(x)$. Since the definition relation of the subsolution for $u_{1}$ actually means $\varphi_{w}^{\prime}\left(u_{1}\right)(w) \leq 0 \forall w$ in $W_{0}^{1, p}(\Omega)$ with $w(x) \geq 0$ almost everywhere (a.e.) on $\Omega$ and that of supersolution for $u_{2}$ is $\varphi_{w}^{\prime}\left(u_{2}\right)(w) \geq 0 \forall w$ in $W_{0}^{1, p}(\Omega)$ verifying $w(x) \geq 0$ a.e. on $\Omega$, we will prove that $v(x)-u_{1}(x) \geq 0$ a.e. on $\Omega$ and $u_{2}(x)-v(x) \geq 0$ a.e. on $\Omega$ using the Gâteaux derivatives of $\varphi$ in $u_{1}$ and $u_{2}$, respectively. $\varphi_{w}^{\prime}\left(u_{1}\right)\left(v-u_{1}\right)=\varphi_{w}^{\prime}\left(u_{1}\right)\left(u_{1}-\right.$ $u)-\varphi_{w}^{\prime}\left(u_{1}\right)(\nabla \varphi(u)) \leq-\varphi_{w}^{\prime}\left(u_{1}\right)(\nabla \varphi(u)) \leq-\varphi_{w}^{\prime}(u)(\nabla \varphi(u))=$ $-\left\|\varphi_{w}^{\prime}(u)\right\|^{2} \leq 0$ (take into account that $u_{1} \leq u, \varphi_{w}^{\prime}\left(u_{1}\right)$ is a linear map and some properties of the metric gradient). Also $\varphi_{w}^{\prime}\left(u_{2}\right)\left(u_{2}-v\right)=\varphi_{w}^{\prime}\left(u_{2}\right)\left(u_{2}-v\right)+\varphi_{w}^{\prime}\left(u_{2}\right)(\nabla \varphi(u)) \geq$ $\varphi_{w}^{\prime}\left(u_{2}\right)(\nabla \varphi(u)) \geq \varphi_{w}^{\prime}(u)(\nabla \varphi(u))=\left\|\varphi_{w}^{\prime}(u)\right\|^{2} \geq 0 . \varphi$ is lower bounded on $\mathscr{F}, \varphi$ being actually continuous (for this see, for instance, [2]). Until now, applying Proposition 5, for every $\left(v_{n}\right)_{n \geq 1}$ a minimizing sequence for $\varphi$ on $\mathscr{F}$, there is a sequence $\left(u_{n}\right)_{n \geq 1}$ in $\mathscr{F}$ such that $\varphi\left(u_{n}\right) \leq \varphi\left(v_{n}\right) \forall n, \lim _{n \rightarrow \infty}\left\|u_{n}-v_{n}\right\|=$ $0, \lim _{n \rightarrow \infty}\left\|\varphi_{w}^{\prime}\left(u_{n}\right)\right\|=0$. So $\lim _{n \rightarrow \infty} \varphi\left(u_{n}\right)=c$ since $c:=$ $\inf \varphi(\mathscr{F})$, we have $\lim _{n \rightarrow \infty}\left\|\varphi_{w}^{\prime}\left(u_{n}\right)\right\|=0$ already, also the last property from $(\mathrm{PS})_{c, \mathscr{F}}$ condition is verified. Finish the proof applying once again Proposition 5.

Example 10. Consider the problem ( $\Omega$ open bounded of $C^{1}$ class in $\mathbf{R}^{N}, N \geq 3$ )

$$
\begin{gathered}
-\Delta_{p} u=\alpha(x) u|u|^{p-2}, \\
u=0 \quad \text { on } \partial \Omega,
\end{gathered}
$$

where $p=2 N /(N-2) ; \alpha$ is continuous with $1 \leq \alpha(x) \leq a<$ $+\infty$ on $\Omega$. Then $u_{1}:=1$ is a weak subsolution, $u_{2}:=M, M>1$ sufficiently big, is a weak supersolution, $|f(x, s)| \leq a|s|^{p-1}$ (condition (17)), and $s \rightarrow \alpha(x) s|s|^{p-2}+s$ is increasing in $s$ on $[1, M]$; consequently, according to Proposition 9 , (32) has a weak solution $\bar{u}$ with $1 \leq \bar{u}(x) \leq M$ a.e. on $\Omega$.

Proposition 11. Let $\Omega$ be an open bounded of $C^{1}$ class set in $\mathbf{R}^{N}, N \geq 3$ and $f: \Omega \times \mathbf{R} \rightarrow \mathbf{R}$ a Carathéodory function and $u_{1}, u_{2}$ from $W_{0}^{1, p}(\Omega)$ bounded weak subsolution and weak supersolution, respectively, of $(* *)$ with $u_{1}(x) \leq u_{2}(x)$ a.e. on $\Omega$. Suppose that $f$ verifies (17) and there is $\rho>0$ such that the function $g: g(x, s)=f(x, s)+\rho s$ is strictly increasing in s on $\left[\inf u_{1}(\Omega), \sup u_{2}(\Omega)\right]$. Then there is a weak solution $\bar{u}$ of $(* *)$ in $W_{0}^{1, p}(\Omega)$ with the property

$$
u_{1}(x) \leq \bar{u}(x) \leq u_{2}(x) \quad \text { a.e. on } \Omega \text {. }
$$

Proof. Follow step by step the above proof for Proposition 9 considering the real reflexive strictly convex Banach space $X=W_{0}^{1, p}(\Omega)$ endowed with the norm $u \rightarrow \mid \mathbf{|} \mathbf{|}=$ $\left(\sum_{i=1}^{N}\left\|\partial u / \partial x_{i}\right\|_{L^{p}(\Omega)}^{p}\right)^{1 / p}$ or the equivalent norm $u \rightarrow|u|_{1, p}=$ $\sum_{i=1}^{N}\left\|\partial u / \partial x_{i}\right\|_{L^{p}(\Omega)}$ which both are also equivalent to the other two norms used at Proposition 9. The function $\varphi$ is from (19) having the weak derivative given in Theorem 7 . Using similar calculus, obtain similar conclusion.

Example 12. Consider the problem ( $\Omega$ open bounded of $C^{1}$ class in $\mathbf{R}^{N}, N \geq 3$ ):

$$
\begin{gathered}
-\Delta_{p}^{s} u=\alpha(x) u|u|^{p-2} \\
u=0 \quad \text { on } \partial \Omega,
\end{gathered}
$$

where $p=2 N /(N-2)$ and $\alpha$ is continuous with $1 \leq \alpha(x) \leq$ $a<+\infty$ on $\Omega$. Then $u_{1}:=1$ is a weak subsolution, $u_{2}:=M$, $M>1$ sufficiently big, is a weak supersolution, $|f(x, s)| \leq$ $a|s|^{p-1}$ (condition (17)), and $s \rightarrow \alpha(x) s|s|^{p-2}+s$ is increasing in $s$ on $[1, M]$; consequently, according to Proposition 11, (34) has a weak solution $\bar{u}$ with $1 \leq \bar{u}(x) \leq M$ a.e. on $\Omega$.

\section{Critical Points for Nondifferentiable Functionals}

The sense of the title actually is "not compulsory differentiable." Start this section with the following.

Definition 13. $x_{0}$ is a critical point (in the sense of Clarke subderivative) for the real function $f$ if $0 \in \partial f\left(x_{0}\right)$. In this case $f\left(x_{0}\right)$ is a critical value (in the sense of Clarke subderivative) for $f$.

To clarify this notion, the Clarke subderivative should be introduced. Let $X$ be a real normed space, $E \subset X, f: E \rightarrow \mathbf{R}$, $x_{0} \in \stackrel{\circ}{E}$, and $v \in X$. We set $f^{0}\left(x_{0} ; v\right):=\overline{\lim }_{x \rightarrow x_{0}, t \rightarrow 0+}((f(x+$ $t v)-f(x)) / t) \cdot f^{0}\left(x_{0} ; v\right)$ is by definition Clarke derivative (or the generalized directional derivative) of the function $f$ at $x_{0}$ in the direction $v$. The functional $\xi$ from $X^{*}$ is by definition Clarke subderivative (or generalized gradient) of $f$ in $x_{0}$ if $f^{0}\left(x_{0} ; v\right) \geq \xi(v) \forall v \in X$. The set of these generalized gradients is designated by $\partial f\left(x_{0}\right)$.

Here it is a generalization at $p$-Laplacian and $p$-pseudoLaplacian of an application from [16] of this concept.

Let $\Omega$ be a bounded domain of $\mathbf{R}^{N}$ with the smooth boundary $\partial \Omega$ (topological boundary). Consider the nonlinear boundary value problems $(*)$ and $(* *)$ where $f: \Omega \times \mathbf{R} \rightarrow$ $\mathbf{R}$ is a measurable function with subcritical growth; that is,

$$
|f(x, s)| \leq a+b|s|^{\sigma} \quad \forall s \in \mathbf{R}, x \in \Omega \text { a.e., }
$$

where $a, b>0,0 \leq \sigma<(N+2) /(N-2)$ for $N>2$ and $\sigma \in[0,+\infty)$ for $N=1$ or $N=2$. 
Set as in [15]

$$
\begin{aligned}
& \underline{f}(x, t)=\varliminf_{s \rightarrow t} f(x, s), \\
& \bar{f}(x, t)=\varlimsup_{s \rightarrow t} f(x, s) .
\end{aligned}
$$

Suppose

$\underline{f}, \bar{f}: \Omega \times \mathbf{R} \longrightarrow \mathbf{R}$ are measurable with respect to $x$.

We emphasize that (II) is verified in the following two cases:

(i) $f$ is independent of $x$.

(ii) $f$ is Baire measurable and $s \rightarrow f(x, s)$ is decreasing $\forall x \in \Omega$, in which case we have

$$
\begin{aligned}
& \bar{f}(x, t)=\max \{f(x, t+), f(x, t-)\}, \\
& \underline{f}(x, t)=\min \{f(x, t+), f(x, t-)\} .
\end{aligned}
$$

Definition 14. $u$ from $W_{0}^{1, p}(\Omega), p>1$ is solution of $(*)$ and $(* *)$, respectively, if $u=0$ on $\partial \Omega$ in the sense of trace and

$$
\begin{aligned}
& -\Delta_{p} u(x) \in[\underline{f}(x, u(x)), \bar{f}(x, u(x))] \quad \text { in } \Omega \text { a.e. } \\
& \text { respectively } \\
& -\Delta_{p}^{s} u(x) \in[\underline{f}(x, u(x)), \bar{f}(x, u(x))] \quad \text { in } \Omega \text { a.e. }
\end{aligned}
$$

Define $W^{1, p}(\Gamma)$ with $p \in(1,+\infty)$, $\Gamma$ regular differential manifold, e.g., $\Gamma=\partial \Omega, \Omega$ open of $C^{1}$ class with $\partial \Omega$ bounded. In this situation, there exists a unique linear continuous operator $\gamma: W^{1, p}(\Omega) \rightarrow W^{1-1 / p, p}(\partial \Omega)$, the trace, such that $\gamma$ is surjective and $u \in W^{1, p}(\Omega) \cap C(\bar{\Omega}) \Rightarrow \gamma(u)=u \mid \partial \Omega$. This gives a sense for $u \mid \partial \Omega$, for any $u$ in $W^{1, p}(\Omega)$. Moreover, $\gamma^{-1}(0)=W_{0}^{1, p}(\Omega)$.

Let be $X:=W_{0}^{1, p}(\Omega)$, but in the first case $(*)$, the norm endowing $X$ is $\|\cdot\|_{1, p}$, that is, $\|u\|_{1, p}=\|u\|_{W_{0}^{1, p}(\Omega)}=$ $\|u\|_{L^{p}(\Omega)}+\sum_{i=1}^{N}\left\|\partial u / \partial x_{i}\right\|_{L^{p}(\Omega)}$, which is equivalent to the norm $u \rightarrow\left(\|u\|_{L^{p}(\Omega)}^{p}+\sum_{i=1}^{N}\left\|\partial u / \partial x_{i}\right\|_{L^{p}(\Omega)}^{p}\right)^{1 / p}$. For the second case $(* *)$, equip the same set $X$ by the norm $u \rightarrow|u|_{1, p}=$ $\left(\sum_{i=1}^{N}\left\|\partial u / \partial x_{i}\right\|_{L^{p}(\Omega)}^{p}\right)^{1 / p}$, which is equivalent to $u \rightarrow|u|_{1, p}=$ $\sum_{i=1}^{N}\left\|\partial u / \partial x_{i}\right\|_{\mathbf{L}^{p}(\Omega)}$. R:

Associate to $(*)$ the locally Lipschitz functional $\Phi: X \rightarrow$

$$
\Phi(u)=\frac{1}{p}\|u\|_{1, p}^{p}-\int_{\Omega} F(x, u) d x, \quad u \in X,
$$

and associate to $(* *)$

$$
\Phi(u)=\frac{1}{p}|\mathbf{l}|_{1, p}^{p}-\int_{\Omega} F(x, u) d x, \quad u \in X,
$$

where $F(x, s)=\int_{0}^{s} f(x, t) d x$. Set

$$
\begin{aligned}
Q(u) & :=\frac{1}{p}\|u\|_{1, p}^{p}, \quad u \in X, \\
\Psi_{1}(u) & :=\int_{\Omega} F(x, u) d x, \quad u \in X, \\
\text { respectively } Q(u) & :=\frac{1}{p}|u|_{1, p}^{p}, \quad u \in X, \\
\Psi_{1}(u) & :=\int_{\Omega} F(x, u) d x, \quad u \in X,
\end{aligned}
$$

$F$, a map defined on $\Omega \times \mathbf{R}$, taking values in $\mathbf{R}$, is locally Lipschitz (use (I)). The functional $\Psi: \mathbf{L}^{\sigma+1}(\Omega) \rightarrow \mathbf{R}$, $\Psi(u)=\int_{\Omega} F(x, u) d x$, is also locally Lipschitz (again (I)). Using Sobolev embedding $X \subset \mathbf{L}^{\sigma+1}(\Omega)$, we obtain that $\Psi_{1}:=\Psi \mid X$ is locally Lipschitz on $X$, which implies $\Phi$ locally Lipschitz on $X$, and consequently, according to a local extremum result for Lipschitz functions (if $x_{0}$ is a point of local extremum for $f$, then $0 \in \partial f\left(x_{0}\right)$ ), the critical points of $\Phi$ for Clarke subderivative can be taken into account. One may state the following.

Proposition 15. Suppose (I) and (II) are satisfied. Then $\Psi$ is locally Lipschitz on $\mathbf{L}^{\sigma+1}(\Omega)$ and

(i) $\partial \Psi(u) \subset[\underline{f}(x, u(x)), \bar{f}(x, u(x))]$ in $\Omega$ a.e,

(ii) if $\Psi_{1}=\Psi \mid X$, where $X=W_{0}^{1, p}(\Omega)$ endowed with the norm $\|\cdot\|_{1, p}$ for the problem $(*)$ and $\mathbf{I} \cdot \mathbf{I}_{1, p}$ for the problem $(* *)$, respectively, then

$$
\partial \Psi_{1}(u) \subset \partial \Psi(u) \quad \forall u \in X
$$

Proof. The proof for (i) can be found in [15], Theorem 2.1, which remained here the same, while the problem was solved for the Laplacian with $X=H_{0}^{1}(\Omega)$ only. In order to prove (ii), use Theorem 2.2 from [15] observing for both cases ( $X$ endowed with each one of those two norms) that $X$ is reflexive and dense in $\mathbf{L}^{\sigma+1}(\Omega)$ as can be seen, for instance, summarized in [2].

Proposition 16. If (I) and (II) are verified, every critical point of $\Phi$ is solution for $(*)$ and $(* *)$, respectively.

Proof.

Problem $(*)$. Let $u_{0}$ be a critical point for $\Phi$. We have

$$
0 \in \partial \Phi\left(u_{0}\right) \subset \partial Q\left(u_{0}\right)+\partial\left(-\Psi_{1}\right)\left(u_{0}\right)
$$

since $\Phi \stackrel{(39)}{=} Q-\Psi_{1}$, and apply some rules of subdifferential calculus concerning finite sums. $\partial Q\left(u_{0}\right)=\left\{Q^{\prime}\left(u_{0}\right)\right\}$, where $Q^{\prime}\left(u_{0}\right)(v)=\int_{\Omega}\left|\nabla u_{0}\right|^{p-2} \cdot \nabla v d x=\left\langle-\Delta_{p} u_{0}, v\right\rangle[2]$.

Using (44) and a specific property of a function $f$ Lipschitz around $x_{0}\left(f^{0}\left(x_{0} ; v\right)=\sup _{\xi \in \partial f\left(x_{0}\right)} \xi(v), \forall v \in X, f^{0}\right.$ the Clarke derivative of $f$ ), we find

$$
0 \leq \int_{\Omega}\left|\nabla u_{0}\right|^{p-2} \cdot \nabla v d x+\left(-\Psi_{1}\right)^{0}\left(u_{0} ; v\right) .
$$


But $\left(-\Psi_{1}\right)^{0}\left(u_{0} ; v\right)=\Psi_{1}^{0}\left(u_{0} ;-v\right)$ (a property of the Clarke derivative, see [1]) and thus

$$
\int_{\Omega}\left|\nabla u_{0}\right|^{p-2} \cdot \nabla(-v) d x \leq \Psi^{0}\left(u_{0} ;-v\right) \quad \forall v \in X
$$

that is,

$$
\mu_{0}(v):=\int_{\Omega}\left|\nabla u_{0}\right|^{p-2} \cdot \nabla v d x \leq \Psi_{1}^{0}\left(u_{0}, v\right) \quad \forall v \in X
$$

$\mu_{0}=-\Delta_{p} u_{0} \in \partial \Psi_{1}\left(u_{0}\right)$ and, using Proposition 15, $-\Delta_{p} u_{0} \in$ $\partial \Psi\left(u_{0}\right)$. Since $\partial \Psi\left(u_{0}\right) \subset\left(\mathbf{L}^{\sigma+1}(\Omega)\right)^{*}=\mathbf{L}^{(\sigma+1) / \sigma}(\Omega)$, it results $u_{0} \in W^{2,(\sigma+1) / \sigma}(\Omega)$ and (37):

$$
-\Delta_{p} u_{0}(x) \in\left[\underline{f}\left(x, u_{0}(x)\right), \bar{f}\left(x, u_{0}(x)\right)\right]
$$

in $\Omega$ a.e.

Problem $(* *)$. Let $u_{0}$ be a critical point for $\Phi$. We have

$$
0 \in \partial \Phi\left(u_{0}\right) \subset \partial Q\left(u_{0}\right)+\partial\left(-\Psi_{1}\right)\left(u_{0}\right)
$$

since $\Phi \stackrel{(40)}{=} Q-\Psi_{1}$, and apply some rules of subdifferential calculus concerning finite sums. $\partial Q\left(u_{0}\right)=\left\{Q^{\prime}\left(u_{0}\right)\right\}$, where $Q^{\prime}\left(u_{0}\right)(v)=\sum_{i=1}^{N} \int_{\Omega}\left|\partial u_{0} / \partial x_{i}\right|^{p-2}\left(\partial u_{0} / \partial x_{i}\right)\left(\partial v / \partial x_{i}\right) d x=$ $\left\langle\Delta_{p}^{s} u_{0}, v\right\rangle[2]$.

Using (49) and a mentioned property of a function $f$ Lipschitz around $x_{0}$, we find

$$
0 \leq \sum_{i=1}^{N} \int_{\Omega}\left|\frac{\partial u_{0}}{\partial x_{i}}\right|^{p-2} \frac{\partial u_{0}}{\partial x_{i}} \frac{\partial v}{\partial x_{i}} d x+\left(-\Psi_{1}\right)^{0}\left(u_{0} ; v\right)
$$

But $\left(-\Psi_{1}\right)^{0}\left(u_{0} ; v\right)=\Psi_{1}^{0}\left(u_{0} ;-v\right)$ (a property of the Clarke derivative, see [1]) and thus

$$
\sum_{i=1}^{N} \int_{\Omega}\left|\frac{\partial u_{0}}{\partial x_{i}}\right|^{p-2} \frac{\partial u_{0}}{\partial x_{i}} \frac{\partial(-v)}{\partial x_{i}} d x \leq \Psi^{0}\left(u_{0} ;-v\right)
$$

$\forall v \in X$

that is,

$$
\mu_{0}(v):=\sum_{i=1}^{N} \int_{\Omega}\left|\frac{\partial u_{0}}{\partial x_{i}}\right|^{p-2} \frac{\partial u_{0}}{\partial x_{i}} \frac{\partial v}{\partial x_{i}} d x \leq \Psi_{1}^{0}\left(u_{0}, v\right)
$$

$\forall v \in X$

$\mu_{0}=-\Delta_{p}^{s} u_{0} \in \partial \Psi_{1}\left(u_{0}\right)$ and, using Proposition 15, $-\Delta_{p}^{s} u_{0} \in$ $\partial \Psi\left(u_{0}\right)$. Since $\partial \Psi\left(u_{0}\right) \subset\left(\mathbf{L}^{\sigma+1}(\Omega)\right)^{*}=\mathbf{L}^{(\sigma+1) / \sigma}(\Omega)$, it results $u_{0} \in W^{2,(\sigma+1) / \sigma}(\Omega)$ and $(38)$ :

$$
-\Delta_{p}^{s} u_{0}(x) \in\left[\underline{f}\left(x, u_{0}(x)\right), \bar{f}\left(x, u_{0}(x)\right)\right] \quad \text { in } \Omega \text { a.e. }
$$

\section{An Application of Ghoussoub-Maurey Linear Principle to $p$-Laplacian and to $p$ - Pseudo-Laplacian}

Start with the statement of this generalized perturbed variational principle.

Theorem 17 (Ghoussoub-Maurey linear principle). Let $X$ be reflexive separable space and $\varphi: X \rightarrow(-\infty,+\infty]$ lower semicontinuous and proper:

(I) If $\varphi$ is bounded from below on the closed bounded nonempty subset $C$, the set

$\left\{\xi \in X^{*}: \varphi+\xi\right.$ strongly exposes $C$ from below $\}$

is of $G_{\delta}$ type and everywhere dense.

(II) If, for any $\xi$ from $X^{*}, \varphi+\xi$ is bounded from below, the set

$\left\{\xi \in X^{*}: \varphi+\xi\right.$ strongly exposes $X$ from below $\}$

is of $G_{\delta}$ type and everywhere dense.

To clarify the involved notions, let $X$ be a real normed space, $f: X \rightarrow(-\infty,+\infty), C$ nonempty subset of $X$, and $x_{0} \in C$. $f$ strongly exposes $C$ from below in $x_{0}$, when $f\left(x_{0}\right)=$ inf $f(C)<+\infty$ and $x_{n} \in C \forall n \geq 1, f\left(x_{n}\right) \rightarrow f\left(x_{0}\right) \Rightarrow x_{n} \rightarrow$ $x_{0}$. " $f$ strongly exposes $C$ from above in $x_{0}$ " has a similar definition. Remark that, taking $C=X$ in the given definition, we fall on the definition of strongly minimum point. And also, a set of $G_{\delta}$ type means a set which is a countable intersection of open sets. A set of $F_{\sigma}$ type means a set which is a countable union of closed sets.

We imply this theorem in two generalizations of a minimization problem of the form [22]

$$
\begin{aligned}
C_{f} & :=\min \left\{\int_{\Omega}\left[\frac{1}{p}\left(|u|^{p}+\sum_{i=1}^{N}\left|\frac{\partial u}{\partial x_{i}}\right|^{p}\right)-f(u)\right] d x:\right. \\
u & \left.\in W_{0}^{1, p}(\Omega),\|u\|_{2^{*}}=1\right\},
\end{aligned}
$$

$$
\begin{aligned}
C_{f} & :=\min \left\{\int_{\Omega}\left(\frac{1}{p} \sum_{i=1}^{N}\left|\frac{\partial u}{\partial x_{i}}\right|^{p}-f(u)\right) d x: u\right. \\
& \left.\in W_{0}^{1, p}(\Omega),\|u\|_{2^{*}}=1\right\},
\end{aligned}
$$

where $\Omega$ is open set of $C^{1}$ class in $\mathbf{R}^{N}, N \geq 3, f \in$ $W^{-1, p^{\prime}}(\Omega)\left(=\left(W_{0}^{1, p}(\Omega)\right)^{*}\right), 1 / p+1 / p^{\prime}=1,2^{*}=2 N /(N-$ 2 ), the critical exponent for the Sobolev embedding (for the necessary explanations, here and in the following, see $[1, I, \S 4$, last section]).

Let $\Omega$ be an open bounded set of $C^{1}$ class in $\mathbf{R}^{N}, N \geq 3$. Consider the problems (*) and (**), where $f: \Omega \times \mathbf{R} \rightarrow \mathbf{R}$ is a Carathéodory function with the growth condition 


$$
\begin{aligned}
& |f(x, s)| \leq c|s|^{p-1}+b(x), \\
& \quad c>0,2 \leq p \leq \frac{2 N}{N-2}, b \in \mathbf{L}^{p^{\prime}}(\Omega), \frac{1}{p}+\frac{1}{p^{\prime}}=1 .
\end{aligned}
$$

The functionals $\varphi: W_{0}^{1, p}(\Omega) \rightarrow \mathbf{R}$,

$$
\begin{aligned}
& \varphi(u) \\
& =\int_{\Omega}\left[\frac{1}{p}\left(|u|^{p}+\sum_{i=1}^{N}\left|\frac{\partial u}{\partial x_{i}}\right|^{p}\right)-F(x, u(x))\right] d x, \\
& \varphi(u)=\int_{\Omega}\left(\frac{1}{p} \sum_{i=1}^{N}\left|\frac{\partial u}{\partial x_{i}}\right|^{p}-F(x, u(x))\right) d x,
\end{aligned}
$$

with $F(x, s):=\int_{0}^{s} f(x, t) d t$, are of $C^{1}$-class Fréchet and their critical points are the weak solutions of the problems $(*)$ and $(* *)$, respectively.

Problem (*). Let $\lambda_{1}$ be the first eigenvalue of $-\Delta_{p}$ in $W_{0}^{1, p}(\Omega)$ with homogeneous boundary condition. We have (see [2, $6.2])$

$$
\lambda_{1}=\inf \left\{\frac{\|u\|_{1, p}^{p}}{|i(u)|_{0, p}^{p}}: u \in W_{0}^{1, p}(\Omega) \backslash\{0\}\right\}
$$

(the Rayleigh - Ritz quotient).

And now give an answer for (56). Use the norm $\|\cdot\|_{p}$ on $W_{0}^{1, p}(\Omega)$ (see above). Denote the dual of $\left(W_{0}^{1, p}(\Omega),\|\cdot\|_{1, p}\right)$ by $W^{-1, p^{\prime}}(\Omega)$, where $p^{\prime}$ is the conjugate of $p$ (i.e., $1 / p+1 / p^{\prime}=1$ ).

Proposition 18. Under the above assumptions and in addition the growth condition

$$
F(x, s) \leq c_{1} \frac{s^{p}}{p}+\alpha(x) s,
$$

with $0<c_{1}<\lambda_{1}, \alpha \in \mathbf{L}^{q^{\prime}}(\Omega)$ for some $2 \leq q \leq 2 N /(N-2)$ and $f(x,-s)=-f(x, s), \forall x$ from $\Omega$, the following assertions hold:

(i) The set of functions $h$ from $W^{-1, p^{\prime}}(\Omega)$, having the property that the functional $\varphi_{h}: W_{0}^{1, p}(\Omega) \rightarrow \mathbf{R}$,

$$
\varphi_{h}(u)=\frac{1}{p}\|u\|_{p}^{p}-\int_{\Omega}(F(x, u(x))+h(u(x))) d x
$$

has in only one point an attained minimum includes a $G_{\delta}$ set everywhere dense.

(ii) The set of functions $h$ from $W^{-1, p^{\prime}}(\Omega)$, having the property

the problem $\begin{cases}-\Delta_{p} u=f(x, u)+h(u) & \text { in } \Omega \\ u=0 & \text { on } \partial \Omega\end{cases}$

has solutions,

includes $a G_{\delta}$ set everywhere dense. (iii) Moreover, if $s \rightarrow f(x, s)$ is increasing, then the set of functions $h$ from $W^{-1, p^{\prime}}(\Omega)$, having the property

the problem $\begin{cases}-\Delta_{p} u=f(x, u)+h(u) & \text { in } \Omega \\ u=0 & \text { on } \partial \Omega\end{cases}$

has a unique solution,

includes $a G_{\delta}$ set everywhere dense.

Remark 19. This is a generalization to $p$-Laplacian and at $W_{0}^{1, p}(\Omega)$ of Theorem 2.13 from [14].

Proof. It is sufficient to justify (i). Consider, for each $h$ from $W^{-1, p^{\prime}}(\Omega)$, the functional $\xi_{h}$ from $W^{-1, p^{\prime}}(\Omega)$ :

$$
\xi_{h}(u)=-\int_{\Omega} h(u(x)) d x .
$$

One may see that $\varphi_{h}=\varphi+\xi_{h}$ (see (59)). Consequently, according to Ghoussoub-Maurey linear principle, (II), if we show that $\varphi_{h}$ is bounded from below for any $h$ from $W^{-1, p^{\prime}}(\Omega)$, then (i) is proven. But, taking into account the Sobolev embedding and (62), we have $\forall u \in W_{0}^{1, p}(\Omega)$ :

$$
\begin{aligned}
\left(\varphi+\xi_{h}\right)(u)= & \frac{1}{p}\|u\|_{1, p}^{p}-\int_{\Omega} F(x, u(x)) d x \\
& -\int_{\Omega} h(u(x)) d x \\
\geq & \frac{1}{p}\|u\|_{1, p}^{p}-c_{1} \int_{\Omega} \frac{|u(x)|^{p}}{p} d x \\
& -\int_{\Omega} \alpha(x) u(x) d x-\int_{\Omega} h(u(x)) d x \\
\geq & \frac{1}{p}\|u\|_{1, p}^{p}-\frac{c_{1}}{\lambda_{1} p}\|u\|_{1, p}^{p}-\|\alpha\|_{q^{\prime}}\|u\|_{q} \\
& -\|h\|_{W^{-1, p^{\prime}}}\|u\|_{1, p} \\
\geq & \frac{1}{p}\left(1-\frac{c_{1}}{\lambda_{1}}\right)\|u\|_{1, p}^{p}-r\|u\|_{1, p} \\
= & \|u\|_{1, p}\left[\frac{1}{p}\left(1-\frac{c_{1}}{\lambda_{1}}\right)\|u\|_{1, p}^{p}-r\right],
\end{aligned}
$$

$r \in \mathbf{R}$, and hence the conclusion since $1-c_{1} / \lambda_{1}>0$. To prove some of these inequalities,

$$
\begin{aligned}
\int_{\Omega} F(x, u(x)) d x & \leq \int_{\Omega}\left(\frac{|u(x)|^{p}}{p}+\alpha(x) u(x)\right) d x \\
& =\frac{1}{p}\|i(u)\|_{0, p}^{p}+\int_{\Omega} \alpha(x) u(x) d x \\
& \leq \frac{1}{p \lambda_{1}}\|u\|_{1, p}^{p}+\|\alpha\|_{0, q^{\prime}}\|u\|_{q} \\
& \leq \frac{1}{p \lambda_{1}}\|u\|_{1, p}^{p}+K\|u\|_{1, p}
\end{aligned}
$$


(see $q$ and properties of Sobolev spaces, e.g., [2]) and $\int_{\Omega} h(u(x)) d x=\langle h, u\rangle \leq\|h\|_{W^{-1, p^{\prime}}}\|u\|_{1, p}$ (the norm of the linear continuous map).

Problem $(* *)$. Let $\lambda_{1}$ be the first eigenvalue of $-\Delta_{p}^{s}$ in $W_{0}^{1, p}(\Omega)$ with homogeneous boundary condition. We have (see $[2,7.2])$

$$
\lambda_{1}=\inf \left\{\frac{|u|_{1, p}^{p}}{|i(u)|_{0, p}^{p}}: u \in W_{0}^{1, p}(\Omega) \backslash\{0\}\right\}
$$

(the Rayleigh - Ritz quotient).

And now give an answer for (57). Use the norm $\mathbf{I} \cdot \mathbf{I}_{p}$ on $W_{0}^{1, p}(\Omega)$ (see above). Denote also the dual of $\left(W_{0}^{1, p}(\Omega), \mathbf{I} \cdot \mathbf{I}_{p}\right)$ by $W^{-1, p^{\prime}}(\Omega)$, where $p^{\prime}$ is the conjugate of $p$ (i.e., $1 / p+1 / p^{\prime}=$ $1)$.

Proposition 20. Under the above assumptions and in addition the growth condition

$$
F(x, s) \leq c_{1} \frac{s^{p}}{p}+\alpha(x) s,
$$

with $0<c_{1}<\lambda_{1}, \alpha \in \mathbf{L}^{q^{\prime}}(\Omega)$ for some $2 \leq q \leq 2 N /(N-2)$ and $f(x,-s)=-f(x, s), \forall x$ from $\Omega$, the following assertions hold.

(i) The set of functions $h$ from $W^{-1, p^{\prime}}(\Omega)$, having the property that the functional $\varphi_{h}: W_{0}^{1, p}(\Omega) \rightarrow \mathbf{R}$,

$\varphi_{h}(u)=\frac{1}{p}|\boldsymbol{u}|_{p}^{p}-\int_{\Omega}(F(x, u(x))+h(u(x))) d x$

has in only one point an attained minimum includes a $G_{\delta}$ set everywhere dense.

(ii) The set of functions $h$ from $W^{-1, p^{\prime}}(\Omega)$, having the property

the problem $\begin{cases}-\Delta_{p}^{s} u=f(x, u)+h(u) & \text { in } \Omega \\ u=0 & \text { on } \partial \Omega\end{cases}$

has solutions,

includes $a G_{\delta}$ set everywhere dense.

(iii) Moreover, if $s \rightarrow f(x, s)$ is increasing, then the set of functions $h$ from $W^{-1, p^{\prime}}(\Omega)$, having the property

the problem $\begin{cases}-\Delta_{p}^{s} u=f(x, u)+h(u) & \text { in } \Omega \\ u=0 & \text { on } \partial \Omega\end{cases}$

has a unique solution,

includes $a G_{\delta}$ set everywhere dense.
Remark 21. This is a generalization to $p$-pseudo-Laplacian and at $W_{0}^{1, p}(\Omega)$ of Theorem 2.13 from [14].

Proof. The proof and the afferent calculus follow step by step those for Proposition 18. One replaces there the norm $\|\cdot\|_{p}$ on $W_{0}^{1, p}(\Omega)$ by the norm $\mathbf{I} \cdot \mathbf{I}_{p}$ and the inequalities and the considerations remain the same.

\section{Conclusions}

Three ways to obtain and/or characterize weak solutions for two problems of mathematical physics equations involving Dirichlet problems for the $p$-Laplacian and the $p$-pseudoLaplacian are developed.

The first sequence of results used the Ekeland variational principle to obtain theoretical propositions which generalize two statements given by Ghoussoub in which the author replaced the real Hilbert space by real reflexive uniformly convex Banach space, and the Fréchet $C^{1}$-class of the goal function by the conditions imposed to this to be lower semicontinuous and Gâteaux differentiable. It is also worthwhile to underline that the Gâteaux differentiability can be replaced by the property of $\beta$-differentiability, $\beta$ being any bornology. These theoretical statements have been used to characterize weak solutions for the $p$-Laplacian and for the $p$-pseudoLaplacian. Some adequate examples were also given.

The second succession of statements establishes results for nondifferentiable functionals using Clarke gradient and other specific notions until their insertion in characterization of weak solutions for Dirichlet problems with the $p$-Laplacian and the $p$-pseudo-Laplacian, respectively, in $W_{0}^{1, p}(\Omega)$.

The last sequence of assertions starts from GhoussoubMaurey linear principle which is used in order to solve some minimization problems. Generalizations of a minimization problem for the Laplacian given by Brezis and Nirenberg have been obtained in conjunction with characterization of weak solutions of Dirichlet problems for the $p$-Laplacian and for the $p$-pseudo-Laplacian.

\section{Competing Interests}

The author declares that they have no competing interests.

\section{References}

[1] I. Meghea, Ekeland Variational Principle with Generalizations and Variants, Old City Publishing, Philadelphia, Pa, USA; Éditions des Archives Contemporaines, Paris, France, 2009.

[2] I. Meghea, "Surjectivité et théorèmes type alternative de Fredholm pour les opérateurs de la forme $J-S$ et quelques Dirichlet problemes," In press.

[3] I. Meghea, "Two solutions for a problem of partial differential equations," UPB Scientific Bulletin, Series A, vol. 72, no. 3, pp. 41-58, 2010.

[4] I. Meghea, "Some results of Fredholm alternative type for operators of $\lambda \mathrm{J}_{\varphi}-\mathrm{S}$ form with applications," U.P.B. Scientific Bulletin, Series A, vol. 72, no. 4, pp. 21-32, 2010. 
[5] I. Meghea, "Weak solutions for the pseudo-Laplacianusing $\Delta_{p}^{s}$ a perturbed variational principle and via surjectivity results," $B S G$ Proceedings, vol. 17, pp. 140-150, 2010.

[6] I. Meghea, "Weak solutions for $p$-Laplacian and for pseudoLaplacian using surjectivity theorems," BSG Proceedings, vol. 18, pp. 67-76, 2011.

[7] N. Amiri and M. Zivari-Rezapour, "Maximization and minimization problems related to $p$-Laplacian equation on a multiply connected domain," Taiwanese Journal of Mathematics, vol. 19, no. 1, pp. 243-252, 2015.

[8] A. El Khalil and M. Ouanan, "Boundary eigencurve problems involving the $p$-Laplacian operator," Electronic Journal of Differential Equations, vol. 78, pp. 1-13, 2008.

[9] N. B. Rhouma and W. Sayeb, "Existence of solutions for the p-Laplacian involving a radon measure," Electronic Journal of Differential Equations, vol. 2012, no. 35, pp. 1-18, 2012.

[10] N. Yoshida, "Forced oscillation criteria for superlinearsublinear elliptic equations via Picone-type inequality," Journal of Mathematical Analysis and Applications, vol. 363, no. 2, pp. 711-717, 2010.

[11] H. Lanchon-Ducauquis, C. Tulita, and C. Meuris, "Modelisation du transfert thermique dans I'He II," in Congres Francais de Thermique (SFT '00), pp. 15-17, Lyon, France, May 2000.

[12] D. Partridge, Numerical modelling of glaciers: moving meshes and data assimilation [Ph.D. thesis], Department of Mathematics and Statistics, University of Reading, 2013.

[13] M. C. Pélissier, Sur Quelques Problèmes Non Linéaires en Glaciologie, Publications Mathèmatiques d'Orsay no. 1110, U.E.R. Mathématique, Université Paris XI, 1975.

[14] N. Ghoussoub, Duality and Perturbation Methods in Critical Point Theory, Cambridge University Press, 1993.

[15] K. C. Chang, "Variational methods for non-differentiable functionals and their applications to partial differential equations," Journal of Mathematical Analysis and Applications, vol. 80, no. 1, pp. 102-129, 1981.

[16] D. G. Costa and J. V. Gonçalves, "Critical point theory for nondifferentiable functionals and applications," Journal of Mathematical Analysis and Applications, vol. 153, no. 2, pp. 470485, 1990.

[17] I. Ekeland, "On the variational principle," Cahiers de Mathématique de la Décision 7217, Université Paris, 1972.

[18] I. Ekeland, "On the variational principle," Journal of Mathematical Analysis and Applications, vol. 47, pp. 324-353, 1974.

[19] G. Dincă, P. Jebelean, and J. Mawhin, Variational and Topological Methods for Dirichlet Problems with p-Laplacian, Université Catholique de Louvain, 1999.

[20] M. M. Vainberg, Variational Methods in the Study of Nonlinear Operators, Holden Day, San Francisco, Calif, USA, 1964.

[21] C. Meghea and I. Meghea, Treatise on Differential Calculus and Integral Calculus for Mathematicians, Physicists, Chemists and Engineers in Ten Volumes, vol. 2 of Éditions des Archives Contemporaines, Paris, Old City, Philadelphia, Pa, USA, 2013.

[22] H. Brezis and L. Nirenberg, "A minimization problem with critical exponent and non-zero data," in Symmetry in Nature, Scuola Norm. Sup. Pisa, pp. 129-140, 1989. 


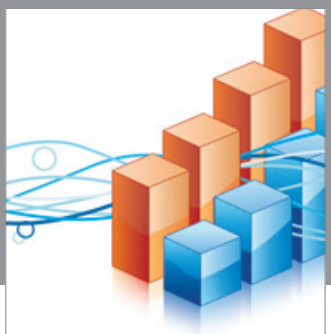

Advances in

Operations Research

vatem alat4

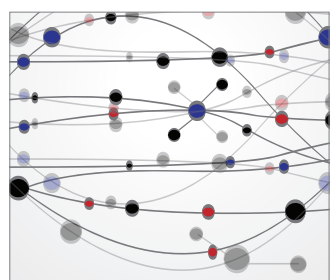

\section{The Scientific} World Journal
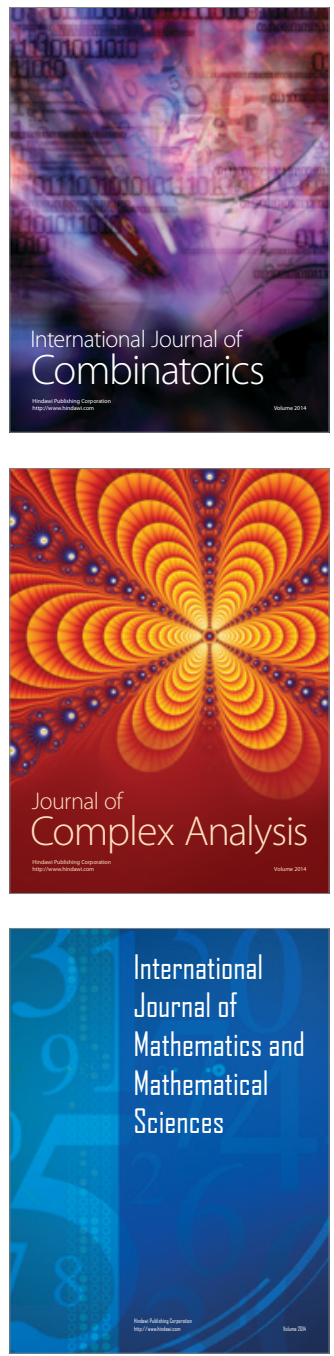
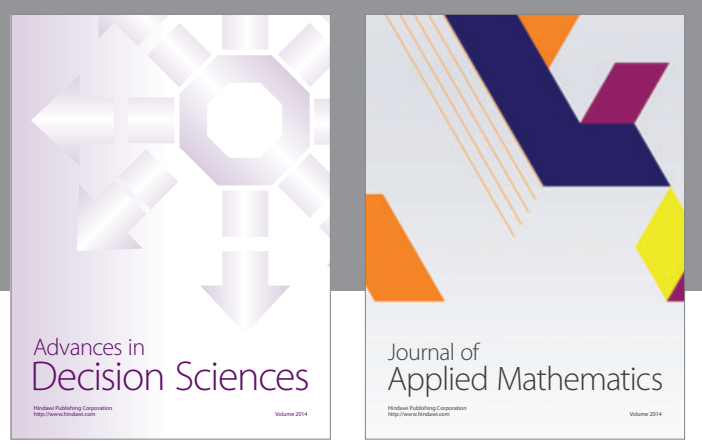

Algebra

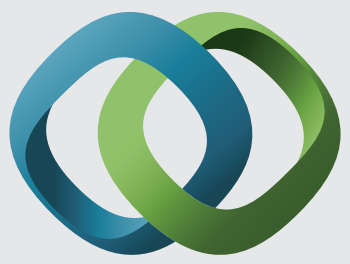

\section{Hindawi}

Submit your manuscripts at

http://www.hindawi.com
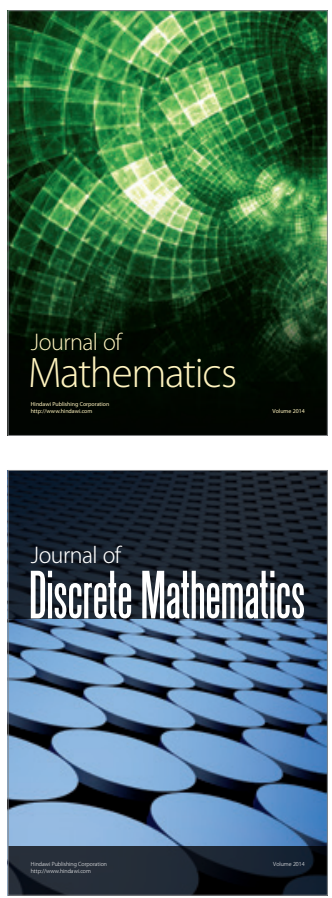

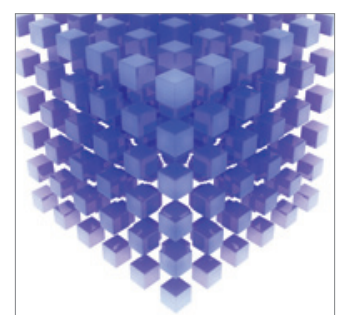

Mathematical Problems in Engineering
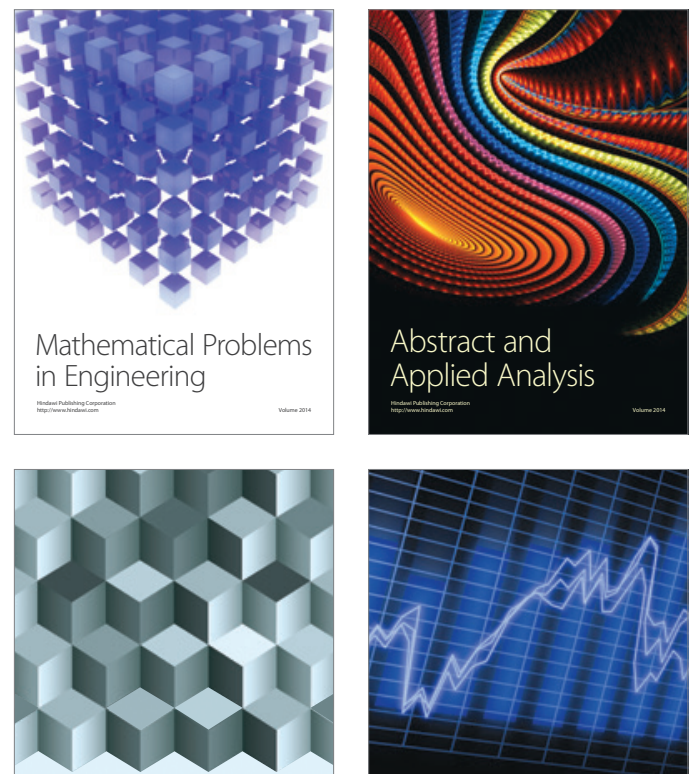

Journal of

Function Spaces

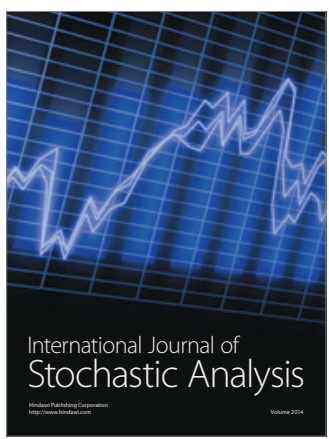

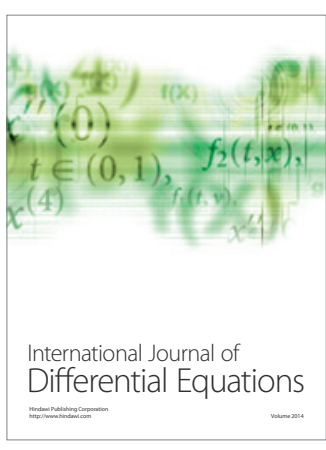
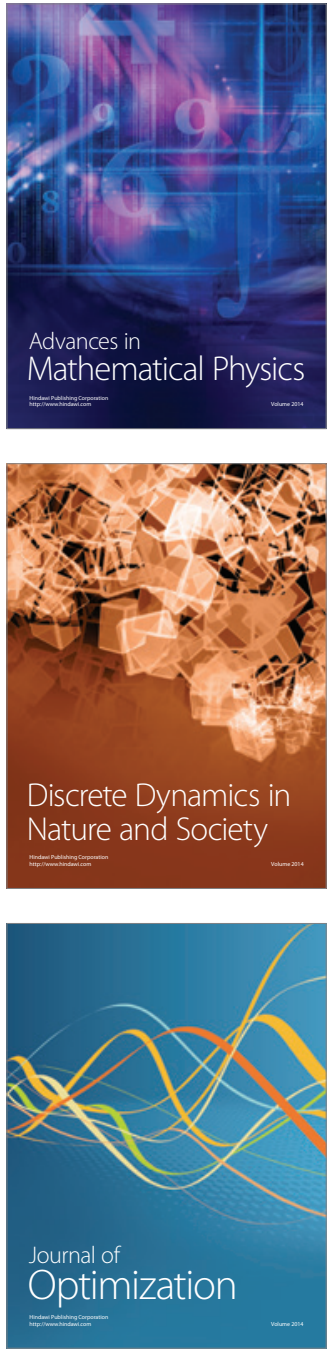